\title{
Pseudo-Electrical Storm in Patients with Implanted Cardioverter Defibrillator
}

\author{
Maurizio Santomauro ${ }^{*}$, Mario Petretta ${ }^{2}$, Carla Riganti ${ }^{3}$, Francesco Elia ${ }^{4}$, Gabriele Iannelli ${ }^{5}$ and Domenico \\ Bonaduce $^{2}$ \\ ${ }^{1,4,5}$ Department of Cardiovascular Emergency, Federico II University, Italy
}

${ }^{2}$ Department of Translational Medical Sciences, Federico II University, Italy

${ }^{3}$ General Direction, Medical School, Federico II University, Italy

*Corresponding author: Maurizio Santomauro, Department of Cardiovascular Emergency, Internal Medicine, Geriatric Medical School, Federico II University, Italy.

Received Date: August 16, 2019

Published Date: August 27, 2019

\begin{abstract}
The Electrical Storm indicates cardiac electrical instability manifested by several episodes of ventricular tachyarrhythmias within a short time. Pseudo-ES is defined as recurrent inappropriate implantable cardioverter-defibrillator discharges over 24 hours. Far from being a minor complication, Pseudo-ES is usually physical and psychological harmful and potentially lethal. The most common causes of inappropriate ICD shock include supraventricular tachycardia with high ventricular response and oversening of peaked T waves or R wave, myopotentials or electrical noise. To approach these patients systematically, it is important to understand that in general, there are four causes of shock. Modern ICD incorporate sophisticated tachycardia detection algorithms within their programming designed to minimize detection mistakes by the device and ICD-related informations. They are often not utilized to their full benefit. Thus, careful attention should be paid the programming of the device. Fine tuning of the detection and differentiation algorithms is critical, and best done by a practitioner who understands the subtle differences among the different manufacturers.
\end{abstract}

Keywords: Implanted cardioverter defibrillator; Pseudo-Electrical Storm; Inappropriate Shock

\section{Background}

Pseudo-Electrical Storm (ES) is defined as recurrent inappropriate implanted cardioverter defibrillator (ICD) discharges over 24 hours. Far from being a minor complication, Pseudo-ES is usually physical and psychological harmful and potentially lethal. The most common causes of inappropriate ICD shock include supraventricular tachycardia with high ventricular response, device oversensing and mechanical malfunctions. Recurrent ICD shocks can cause myocardial injury by direct electrocution cell injury and by activation of signaling pathways in the molecular cascade of heart failure (HF), the most important of all being adrenergic neurohormonal system. Adrenergic operativity may then synergize with recurrent ventricular arrhythmias in exacerbating ventricular dysfunction and worsening HF. Sweeney et al. [1] demonstrated that electrical shocks were associated with an increased risk of death independently of underlying ventricular arrhythmia. Authors esteemed that for every delivered shock, whether appropriate or not, the risk of death increases by $20 \%$. On the other hand, no increased risk was associated with antitachycardia pacing (ATP) therapies. Pseudo-ES does not only cause myocardial damage, but can deplete a full device battery within hours, potentially leaving the patient unprotected from life-threatening arrhythmic events. Pseudo-ES should be treated by immediate intervention to suppress ICD shocks. Moreover, inappropriate discharges from ICD should be avoided at all cost by an optimal device programming.

\section{Inappropriate therapies due to Supraventricular tachycardia}

Inappropriate shocks are one of the main issues to be avoided as they cause patient discomfort, are potentially proarrhythmic, and reduce battery life. The two main causes of inappropriate shock are failure in discriminating supraventricular arrhythmias (SVA) [2-13]. Frequently SVA is associated with a fast-ventricular response leading ventricular rate to fall into VT/VF detection zone causing inappropriate therapy release. This problem occurs more frequently with single-chamber ICD that do not have atrial sensing 
capabilities. Current guidelines do not provide a clear stepwise approach to managing patients at high risk for recurrent shock. Appropriate diagnosis and treatment are critical. To approach these patients systematically, it is important to understand that in general, there are four causes of shock. ICD use a variety of algorithms to discriminate SVA from VT: Atrio ventricular rate comparison, Onset, Stability, Morphology, Rate duration. Modern ICD incorporate sophisticated tachycardia detection algorithms within their programming designed to minimize detection mistakes by the device for reducing inappropriate therapies (SMART by Biotronik*, RHYTM ID by Boston Scientific **, PR Logic by Medtronic ***, PARAD+Rhythm DiScrimination by Sorin ****, Rate Branch by St Jude*****). Fine tuning of the detection and differentiation algorithms is critical, and best done by a practitioner who understands the subtle differences among the different manufacturers.

\section{Inappropriate Shock due to Oversensing}

Signal misinterpretation is the other big deal leading to inappropriate shocks. It may depend on some programmed variables; easily editable, external, and farfield interferences; or lead failure that usually requires an interventional approach [2-13] Major ones are listed below: -T wave oversensing: it happens when a high amplitude $\mathrm{T}$ wave is erroneously recognized as an $\mathrm{R}$ wave. It may happen because of the low ventricular sensing threshold necessary to recognize even low-amplitude VF. This problem can be solved by increasing sensing threshold, lengthening refractory period, or changing sensing decay parameters to suppress $\mathrm{T}$ wave detection. - Double-counted R waves: may occur as a result of local ventricular delay in the baseline state or conduction delay caused by drugs or electrolyte abnormalities. It may also occur in patients with a double or triple lead ICD, long PR interval and loss of RV pacing capture. The ICD may count both the paced ventricular event and the spontaneous $\mathrm{R}$ wave conducted from the atrium. Finally, another common cause of double counting is loss of RV capture in cardiac resynchronization therapy-defibrillator (CRT-D). The device counts both the paced ventricular event and the RV depolarization originating from the LV lead. R-wave double counting results in alternation of 2 ventricular cycle lengths. The second component of the $\mathrm{R}$ wave is usually sensed as soon as the blanking period terminates and is always classified in the VF zone. The classification of the first one depends on the programming of the techy zones and on the heart rate. The double counting can manifest during sinus rhythm, only during precocious ventricular complex (PVC) or during slow VT with a misclassification as VF, the true rate being overestimated and possibly leading to shocks. Prolongation of the ventricular blanking period from the nominal value corrects ventricular double counting in the majority of cases and must be proposed as the first step when possible, keeping in mind that a common concern is true VF undersensing when the blanking period is over-extended. Similarly, decreasing the programmed ventricular sensitivity may resolve the problem in a certain number of cases but this option requires that reliable sensing of VF is confirmed at the reduced level of sensitivity.
Moreover, lowering ventricular sensitivity may be dangerous and useless since the amplitude of the 2 signals may be as high. Programming of very high VF zone to solve the problem seems also inappropriate. Atrial far-field sensing: inappropriately detecting an atrial paced event in the ventricular chamber related to the sensing of events from one chamber in another chamber. Cross-chamber blanking periods are an integral part of the ICD and CRT-D sensing systems. They are used to suppress detection of device-generated artifact as well as certain intrinsic signal artifacts. Events that occur during refractory and cross-chamber blanking periods are ignored for the purposes of pacing timing cycles and ventricular tachycardia detection. Each refractory and fixed cross-chamber blanking period includes a re-triggerable noise window, which helps to detect and classify persistent noise. Cross-chamber blanking periods are designed to promote appropriate sensing of in chamber events and prevent oversensing of activity in another chamber. Crosschamber blanking periods are initiated by paced and/or sensed events in an adjacent chamber. To promote continuous pacing for pacemaker dependent patients, it may be preferable to lessen the potential for ventricular oversensing of atrial paced events by programming a longer blanking period, even though the likelihood of undersensing a PVC may increase (should it occur within the RVBlank after A-Pace cross-chamber blanking period). For patients with sick sinus syndrome and frequent PVC who are not pacemaker dependent, it may be preferable to shorten the blanking period to lessen the potential for undersensing a PVC, even though the likelihood for ventricular oversensing of an atrial paced event may increase. Residual energy on the defibrillation lead after shock delivery can increase the likelihood of crosstalk / far-field sensing. As this residual energy dissipates with time after shock delivery, the potential for crosstalk / far-field sensing also decreases. To reduce oversensing after shock delivery, a longer fixed value is automatically applied for all cross-blanking periods during the PostTherapy Period. Electromagnetic interference (EMI): is fortunately fairly infrequent with bipolar leads, but still occurs. There are many causes of EMI, the most common of which include arc welding, improper copper wiring in a shower, carrying stereo speakers, working on a running car engine, lingering in a store's surveillance gating, radiofrequency transmitting devices, automobile ignition systems, radar, and portable music players. To prevent shock from EMI often involves a certain amount of detective work. Once the cause of the EMI is identified, the patient must avoid the culprit, or in some cases, the device can be reprogrammed to prevent recognition of the EMI [14-16]. -Pectoral Myopotentials: farfield myopotential recording may lead to inappropriate arrhythmic detection. This problem occurred in the past with unipolar leads using large sensing fields and is now largely avoided with the modern bipolar leads, recording more localized signals only. These high-frequency, variable amplitude signals are prominent on electrograms that include the ICD can, including shock electrograms and leadless ECG. They may be reproduced by pectoral muscle exercise. However, because ICD do not use these signals as primary sensing channels, pectoral myopotentials do not cause oversensing if the lead is intact. However, they may cause misclassification of 
exercise-induced sinus tachycardia as VT because algorithms that discriminate VT from SVT based on ventricular electrogram morphology use the RV coil-can vector as the default signal. Pectoral myopotentials might also interfere with algorithms that evaluate lead integrity by comparing near-field and far-field signals [14]; -Diaphragmatic Myopotentials: these low-amplitude, highfrequency signals are more prominent on the sensing electrogram than the shock electrogram because the sensing bipole is closer to the source. Their amplitude varies with respiration, but not the cardiac cycle. Oversensing is most common with integrated bipolar sensing at the RV apex and rare with dedicated bipolar sensing or leads in the RV outflow tract. It occurs when sensitivity is maximal, after long diastolic intervals or ventricular paced events, and often ends with a sensed R wave, which reduces sensitivity abruptly. Thus, it commonly occurs in pacemaker-dependent patients, in whom inhibition of pacing maintains high ventricular sensitivity, resulting in persistent oversensing and inappropriate detection of VF. It may present as syncope because of inhibition of pacing followed by an inappropriate shock. Oversensing may be reproduced by monitoring real-time electrograms during deep breathing or straining in different positions, after programming VF detection off $[14,15]$-Lead failure: has many causes, but some of the most common include fractured leads, dislodged leads, loss of capture after ICD shock, redundant loops of endocardial leads, chatter in active fixation lead, loose set screew or adapter. Management of this category of shock involves fixing the implanted system, either with device reprogramming or reoperation. In these cases lead extraction and/or new lead insertion is the only choice. Modern devices usually provide alerts for lead integrity [16].

\section{Device Reprogramming}

Optimization of ICD programming in order to avoid unnecessary shock is mandatory in patients experiencing Pseudo-ES. As stated above, arrhythmic detection and treatment by ICD is a step process including several variables such as heart rate threshold, number of intervals to detect, discrimination of SVA, and type and number of therapies released. Each of these steps can be tailored upon patient characteristics to avoid unnecessary treatment. A patient who receives multiple shocks is not difficult to identify by ispecting data stored in the ICD. At that point in time, it is critical to define the etiology of the shocks. Perform initial evaluation as above. The device needs to be fully interrogated, with careful analysis of all of the stored electrocardiograms (EGM) recorded from the recent therapies and performing specific troubleshooting. Display and analysis of real-time and stored electrograms (EGMs) on a programmer can be a valuable tool during an implant procedure or a post-implant follow-up. EGMs can be used to assess sensing and capture, and to evaluate lead system integrity. Evaluation of all active leads can be performed to assist with patient assessment and device programming, as well as identify possible lead or lead connection issues. The EGM evaluation tools facilitate review of device and patient information, including: -ability to measure amplitude of cardiac signals, -ability to measure time between cardiac signals, -selectable gain for each channel. When evaluating lead signals: the signals from the implanted leads should be continuous and without artifact, similar to a surface ECG. A discontinuous signal may indicate a poor lead connection or damaged lead that may necessitate lead replacement. Inadequate signals may result in failure of the device system to properly detect an arrhythmia, inability to deliver programmed therapy, or unnecessary delivery of therapy. The single most important diagnostic test is interrogation of the patient's device. If device malfunction is suspected, therapy can be immediately suspended by placing a magnet over the ICD can Figure 1.

Unlike a pacemaker, this will not alter the device's pacing capabilities Table 1.

Should a true ventricular arrhythmia subsequently declare itself, removing the magnet will immediately reactivate all device therapies. Figure 1 shows various ICD responses to clinical magnet application. ICD models from St. Jude Medical and Boston Scientific, however, have additional programmable features to ignore the magnet or respond differently to a magnet. All biventricular ICD behave like any other ICD of the corresponding manufacturer. In most cases, anti-tachycardia therapy resumes with removal of the magnet [17]. However, in some instances, magnet removal may or may not re-enable anti-tachycardia therapy. Specific care should be exercised in Boston ICD since some of the older models are equipped with circuitry that enables the magnet to permanently Programme the anti-tachycardia therapy to 'OFF'. In addition, magnet application on Sorin ICD changes the pacing rate without altering the pacing mode. Rate response of ICD is not influenced by magnet application. Only Lumax® ICD model (Biotronik) the anti-tachycardia therapy is disabled only for $8 \mathrm{~h}$ on constant magnet application, after which the ICD automatically resumes anti-tachycardia therapy. All clinical magnet should by used in different manufacturers and models. The site of magnet placement is important because a poorly positioned magnet may not produce the desired effects Figure 1.

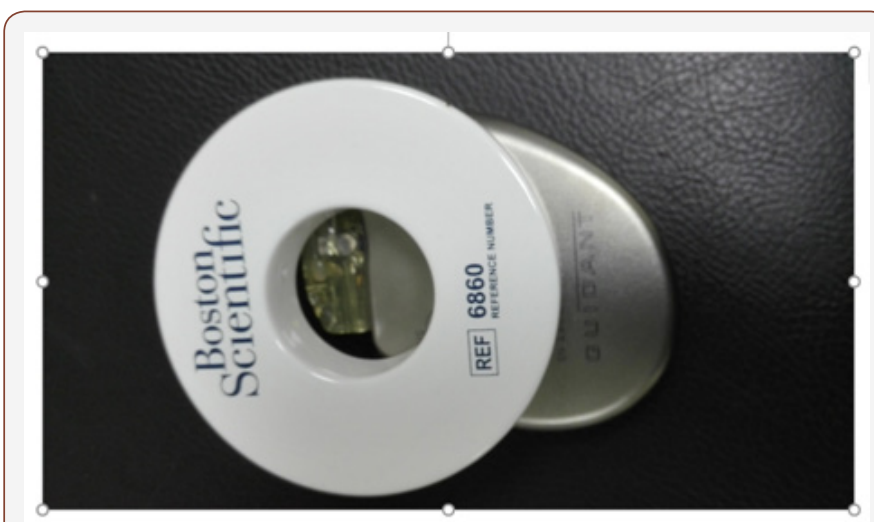

Figure 1: The correct site placement of magnet.

In patients with subcutaneous (S-ICD) System if an programmer is not available, a magnet may be used to prevent arrhythmia detection and/or shock delivery by an S-ICD. Therapy will only be temporarily inhibited during magnet application; a magnet cannot 
be used to program therapy OFF. If the magnet is correctly placed over the device, beeping tones ( $\mathrm{r}$-wave synchronous) will be heard approximately one second after the magnet is applied. Arrhythmia detection is now suspended, and shock therapy is inhibited. Therapy remains inhibited for as long as the magnet remains correctly positioned Table 1 .

Table 1: Response to magnet from various manufacturers ICD.

\begin{tabular}{|c|c|c|}
\hline Manufacturers & $\begin{array}{l}\text { Tachyarrhythmia } \\
\text { Therapies }\end{array}$ & $\begin{array}{l}\text { Bradycardia } \\
\text { Therapies }\end{array}$ \\
\hline Biotronik & $\begin{array}{l}\text { Transient inhibition of } \\
\text { detection And therapy } \\
\text { delivery for VT/VF, } \\
\text { lasting as long as the } \\
\text { magnet is positioned } \\
\text { over the ICD No beeping } \\
\text { tones }\end{array}$ & No change the pacing \\
\hline Boston & $\begin{array}{l}\text { Transient inhibition of } \\
\text { detection And therapy } \\
\text { delivery for VT/VF, } \\
\text { lasting as long as the } \\
\text { magnet is positioned over } \\
\text { the ICD. If the "change } \\
\text { techy mode with magnet" } \\
\text { function is enabled, } \\
\text { magnet application for } \\
\text { more than } 30 \text { sec results } \\
\text { in deactivation of the } \\
\text { device. Beeping tones }\end{array}$ & No change the pacing \\
\hline Boston sICD & $\begin{array}{l}\text { Transient inhibition of } \\
\text { detection And therapy } \\
\text { delivery for VT/VF, } \\
\text { lasting as long as the } \\
\text { magnet is positioned } \\
\text { over the ICD Beeping } \\
\text { tones }\end{array}$ & No pacing available \\
\hline Medtronic & $\begin{array}{l}\text { Transient inhibition of } \\
\text { detection And therapy } \\
\text { delivery for VT/VF, } \\
\text { lasting as long as the } \\
\text { magnet is positioned } \\
\text { over the ICD Beeping } \\
\text { tones }\end{array}$ & No change the pacing \\
\hline Sorin & $\begin{array}{l}\text { Transient inhibition of } \\
\text { detection And therapy } \\
\text { delivery for VT/VF, } \\
\text { lasting as long as the } \\
\text { magnet is positioned } \\
\text { over the ICD No beeping } \\
\text { tones }\end{array}$ & No change the pacing \\
\hline St Jude- Abbott & $\begin{array}{l}\text { Transient inhibition of } \\
\text { detection And therapy } \\
\text { delivered for VT/VF } \\
\text { lasting as long as the } \\
\text { magnet is positioned } \\
\text { over the ICD No beeping } \\
\text { tones }\end{array}$ & No change the pacing \\
\hline
\end{tabular}

After 60 seconds, the beeping stops, but therapy continues to be inhibited unless the magnet has been moved. No ATP or pacing available. Device return to normal function once magnet is removed.

\section{Conclusion}

ICD is a medical breakthrough but has a complex function. Adequate therapy prolongs survival but unnecessary therapy increases morbidity and mortality. Appropriate function required adequate mechanical component (lead), electrical function (battery and capacitor) software functioning (detection algorithms) and substrate (appropriate VT and safety margin). Pseudo-ES are a life-threatening syndrome and the appropriateness of acute management determines the patient's survival.

\section{Acknowledgment}

The authors thank Ing Luigi Campanile (Boston Team) for his technical assistance for this manuscript

\section{Conflict of Interest}

No conflict of interest.

\section{References}

1. Sweeney MO, Sherfesee L, DeGroot PJ, Wathen MS, Wilkoff BL, et al. (2010) Differences in effects of electrical therapy type for ventricular arrhythmias on mortality in implantable cardioverter defibrillator patients. Heart Rhythm 7(3): 353-360.

2. Cao J, Gillberg JM, Swerdlow CD (2012) A fully automatic, implantable cardioverter-defibrillator algorithm to prevent inappropriate detection of ventricular tachycardia or fibrillation due to T-wave oversensing in spontaneous rhythm. Heart Rhythm 9(4): 522-530.

3. Wilkoff BL, Williamson BD, Stern RS, Moore SL, Lu F, et al. (2008) Strategic programming of detection and therapy parameters in implantable cardioverter-defibrillators reduces shocks in primary prevention patients. J Am Coll Cardiol 52(7): 541-550.

4. Tan VH, Wilton SB, Kuriachan V, Sumner GL, Exner DV, et al. (2014) Impact of programming strategies aimed at reducing nonessential implantable cardioverter defi brillator therapies on mortality: a systematic review and meta-analysis. Circ Arrhythm Electrophysiol 7(1): 164-170.

5. Gasparini M, Proclemer A, Klersy C, Kloppe A, Lunati M, et al (2013) Effect of long-detection interval vs standard detection interval for implantable cardioverter-defi brillators on antitachycardia pacing and shock delivery: the ADVANCE III randomized clinical trial. JAMA 309(18): 1903-1911.

6. Madhavan M, Friedman PA (2013) Optimal programming of implantable cardiac-defibrillators. Circulation 128(6): 659-672.

7. Moss AJ, Schuger C, Beck CA, Brown MW, Cannom DS, et al. (2012) Reduction in Inappropriate Therapy and Mortality through ICD Programming. N Engl J Med 367(24): 2275-2283.

8. Banker R, Mitchell R, Badhwar N, Goldschlager N (2010) Pacemaker and Implantable Cardioverter-Defibrillator Emergencies, Cardiac Intensive Care (Second Edition) Chapter 26, Elservier Inc Pp. 310-338.

9. Auricchio A, Schloss EJ, Kurita T, Meijer A, Gerritse B, et al. (2015) Low inappropriate shock rates in patients with single- and dual/triplechamber implantable cardioverter-defibrillators using a novel suite of detection algorithms: Pain Free SST trial primary results. Heart Rhythm 12(5): 926-936.

10. Saeed M, Hanna I, Robotis D, Styperek R, Polosajian L, et al. (2014) Programming implantable cardioverter-defibrillators in patients with primary prevention indication to prolong time to first shock: results from the PROVIDE study. J Cardiovasc Electrophysiol 25(1): 52-59.

11. Scott PA, Silberbauer J, McDonagh TA, Murgatroyd FD (2014) Impact of prolonged implantable cardioverter-defibrillator arrhythmia detection times on outcomes: a meta-analysis. Heart Rhythm 11(5): 828-835.

12. Peterson PN, Greenlee RT, Go AS, Magid DJ, Cassidy-Bushrow A, et al. (2017) Comparison of Inappropriate Shocks and Other Health Outcomes Between Single- and Dual-Chamber Implantable CardioverterDefibrillators for Primary Prevention of Sudden Cardiac Death: Results From the Cardiovascular Research Network Longitudinal Study of Implantable. Cardioverter-Defibrillators J Am Heart Assoc 6(11): e006937.

13. Ruiz-Granell R, Dovellini EV, Dompnier A, Khalighi K, García-Campo E, et al (2019) Algorithm-based reduction of inappropriate defibrillator shock: Results of the Inappropriate Shock Reduction with PARAD+ 
Rhythm DiScrimination-Implantable Cardioverter Defibrillator Study. Heart Rhythm in press.

14. Mozes A, DeNofrio D, Pham DT, Homoud MK (2011) Inappropriate implantable cardioverter-defibrillator therapy due to electromagnetic interference in patient with a Heart Ware HVAD left ventricular assist device. Heart Rhythm 8(5): 778-780.

15. Swerdlow CD, Asirvatham SJ, Ellenbogen KA, Friedman PA (2014) Troubleshooting Implanted Cardioverter Defibrillator Sensing Problems I. Circulation: Arrhythmia and Electrophysiology 7(6): 1237-1261.
16. Powell BD, Asirvatham SJ, Perschbacher DL, Jones PW, Cha YM, et al (2012) Noise, artifact, and oversensing related inappropriate ICD shock evaluation: ALTITUDE noise study. Pacing Clin Electrophysiol 35(7): 863-869.

17. Jacob S, Panaich SS, Maheshwari R, Haddad JW, Padanilam BJ, et al. (2011) Clinical applications of magnets on cardiac rhythm management devices. EP Europace 13(9): 1222-1230. 